\title{
Targeted RNA-Seq Reveals the M. tuberculosis Transcriptome from an In Vivo Infection Model
}

\author{
Fernanda Cornejo-Granados ${ }^{1}$ (D), Gamaliel López-Leal ${ }^{1}$ (D), Dulce A. Mata-Espinosa ${ }^{2}$, Jorge Barrios-Payán ${ }^{2}$, \\ Brenda Marquina-Castillo ${ }^{2}$, Edgar Equihua-Medina 1, Zyanya L. Zatarain-Barrón ${ }^{2}$, Camilo Molina-Romero ${ }^{2}$, \\ Rogelio Hernández-Pando ${ }^{2, *}$ and Adrian Ochoa-Leyva ${ }^{1, *}$
}

Citation: Cornejo-Granados, F.; López-Leal, G.; Mata-Espinosa, D.A.; Barrios-Payán, J.; Marquina-Castillo, B.; Equihua-Medina, E.; Zatarain-Barrón, Z.L.; Molina-Romero, C.;

Hernández-Pando, R.; Ochoa-Leyva, A. Targeted RNA-Seq Reveals the M. tuberculosis Transcriptome from an In Vivo Infection Model. Biology 2021, 10, 848. https://doi.org/10.3390/ biology10090848

Academic Editor: Wito Richter

Received: 29 June 2021

Accepted: 16 August 2021

Published: 31 August 2021

Publisher's Note: MDPI stays neutral with regard to jurisdictional claims in published maps and institutional affiliations.

Copyright: (C) 2021 by the authors. Licensee MDPI, Basel, Switzerland. This article is an open access article distributed under the terms and conditions of the Creative Commons Attribution (CC BY) license (https:// creativecommons.org/licenses/by/ $4.0 /)$
1 Departamento de Microbiología Molecular, Instituto de Biotecnología, Universidad Nacional Autonóma de México, Cuernavaca 62210, Mexico; fernanda.cornejo@ibt.unam.mx (F.C.-G.); gamlopez@ccg.unam.mx (G.L.-L.); edgar.equihua@uaem.edu.mx (E.E.-M.)

2 Sección de Patología Experimental, Departamento de Patología, Instituto Nacional de Ciencias Médicas y Nutrición “Salvador Zubirán", Vasco de Quiroga 15, Tlalpan, Sección XVI, Ciudad de México 14000, Mexico; dulmat@comunidad.unam.mx (D.A.M.-E.); jorge.barriosp@incmnsz.mx (J.B.-P.); brenda.marquina@comunidad.unam.mx (B.M.-C.); zyanyal@comunidad.unam.mx (Z.L.Z.-B.); camilomol@comunidad.unam.mx (C.M.-R.)

* Correspondence: rogelio.hernandezp@incmnsz.mx (R.H.-P.); adrian.ochoa@ibt.unam.mx (A.O.-L.)

Simple Summary: High-throughput sequencing techniques such as RNA-seq allow a more detailed characterization of the gene expression profile during in vivo infections. However, using this strategy for intracellular pathogens such as Mycobacterium tuberculosis (Mtb) entails technical limitations. Some authors have resorted to flow cytometers to separate infected cells or significantly increase sequencing depth to obtain pathogens' gene expression. However, these options carry additional expenses in specialized equipment. We propose an experimental protocol based on differential cell lysis and a probe-based ribosomal depletion to determine the gene expression of Mtb and its host during in vivo infection. This method allowed us to increase the number of observed expressed genes from 13 using a traditional RNA-seq approach to 702. In addition, we observed the expression of genes essential for establishing the infection, codifying proteins such as PE-PGRS, lipoproteins lppN and LpqH, and three ncRNAs (small RNA MTS2823, transfer-messenger RNA RF00023, and ribozyme RF00010). We believe our method represents a valuable alternative to current RNA-seq approaches to study host-pathogen interactions and will help explore host-pathogen mechanisms in tuberculosis and other similar models of intracellular infections.

Abstract: The study of host-pathogen interactions using in vivo models with intracellular pathogens like Mycobacterium tuberculosis (Mtb) entails technical limitations, such as: (i) Selecting an efficient differential lysis system to enrich the pathogen cells; (ii) obtaining sufficient high-quality RNA; and (iii) achieving an efficient rRNA depletion. Thus, some authors had used flow cytometers to separate infected cells or significantly increase the sequencing depth of host-pathogen RNA libraries to observe the pathogens' gene expression. However, these options carry additional expenses in specialized equipment typically not available for all laboratories. Here, we propose an experimental protocol involving differential cell lysis and a probe-based ribosomal depletion to determine the gene expression of $\mathrm{Mtb}$ and its host during in vivo infection. This method increased the number of observed pathogen-expressed genes from 13 using the traditional RNA-seq approach to 702. After eliminating rRNA reads, we observed that $61.59 \%$ of $\mathrm{Mtb}$ sequences represented 702 genes, while $38.41 \%$ represented intergenic regions. Some of the most expressed genes codified for IS1081 (Rv2512c) transposase and eight PE-PGRS members, such as PGRS49 and PGRS50. As expected, a critical percent of the expressed genes codified for secreted proteins essential for infection, such as PE68, lppN, and LpqH. Moreover, three Mtb ncRNAs were highly expressed (small RNA MTS2823, transfer-messenger RNA RF00023, and ribozyme RF00010). Many of the host-expressed genes were related to the inflammation process and the expression of surfactant proteins such as the Sftpa and Sftpc, known to bind Mtb to alveolar macrophages and mi638, a microRNA with no previous associations with pulmonary diseases. The main objective of this study is to present the method, and 
a general catalog of the Mtb expressed genes at one point of the in vivo infection. We believe our method represents a different approach to the existing ones to study host-pathogen interactions in tuberculosis and other similar intracellular infections, without the necessity of specialized equipment.

Keywords: transcriptome; tuberculosis; host-pathogen; RNA-seq; in vivo infection

\section{Introduction}

RNA-seq approaches have helped to define the finely regulated host-pathogen interactions. However, its application for in vivo models of intracellular bacteria such as M. tuberculosis (Mtb) entails several limitations: (i) Selecting an efficient cell lysis system [1]; (ii) obtaining sufficient high-quality RNA [1,2]; and (iii) achieving an efficient rRNA depletion [1,2]. Hence, some studies have used infected cell cultures [3,4], labeled bacteria to separate infected from non-infected cells $[1,5]$, increased the number of infecting bacilli to obtain enough RNA [6], or selectively amplified bacterial RNA/cDNA [7]. However, these strategies simplify the myriad factors involved in an in vivo model or modify the gene expression profile by altering the typical course of infection.

Currently, two studies describe the Mtb transcriptome during in vivo murine infection. The first [6] used DNA microarrays to compare the in vivo gene expression in BALB/c vs. $\mathrm{BALB} / \mathrm{c} \mathrm{SCID} / \mathrm{SCID}$ vs. bacterial cultures at different time points of infection. The second [5] compared the bacteria transcriptome of Mtb Erdman marked with fluorescent reporter mCherry between alveolar and interstitial macrophages isolated directly from infected mouse lungs at day 14 post-infection using RNA-seq.

Undoubtedly, the use of RNA-seq offers a more sensitive and quantitative approach to determine the expression of genes and intergenic regions. However, the use of specialized equipment to separate infected from non-infected cells or significantly increasing the sequencing depth to enrich the information on the pathogens' gene expression entails additional expenses not available for all research laboratories worldwide. Overall, the purpose of this study is to present the efficiency and reproducibility of our experimental method to increase the number of observed pathogen expressed genes during tuberculosis in vivo infection. This method could be used in the future to explore the pathogen gene expression profile at different time points of the infection and use different Mtb-infecting strains.

Here, we used a well-characterized murine model for pulmonary tuberculosis $[8,9]$ to obtain the $\mathrm{Mtb}$ and mouse transcriptome from lungs 21-days post-infection using an experimental approach involving differential cell lysis and a probe-based ribosomal depletion.

This murine model is based on the intratracheal instillation of live Mtb bacilli into male BALB/c mice and shows the evolution of the disease in two clear phases, an acute phase that spans from day 1-28 post-infection, characterized by inflammatory infiltrate and formation of granulomas, and an advance phase, from day 28 onwards, characterized by pneumonia, focal necrosis, and fibrosis [9]. Furthermore, we used male mice because of the essential differences in their immune response compared to female mice [10]. Female mice show a hyper-inflammatory response and better protection against tuberculosis, while male mice show anti-inflammatory responses probably favored by testosterone, and are more susceptible to infection.

\section{Materials and Methods}

\subsection{Bacteria Cultures}

The M. tuberculosis (Mtb) H37Rv strain was cultured in Middlebrook 7H9 broth (Millipore, Burlington, MA, USA, Cat. M0178) enriched with ADC Growth Supplement (Millipore, Burlington, MA, USA, Cat. M0553) at $37^{\circ} \mathrm{C}$. The optical density (OD) was monitored weekly, and the purity was assessed with a Zihel-Neelsen stain. As soon as the culture reached the mid-logarithmic phase $(\mathrm{OD}=0.6)$, bacilli were harvested and aliquoted, adjusting to $2.5 \times 10^{5}$ colony-forming units (CFU) in $100 \mu \mathrm{L}$ of phosphate-buffered saline 
(PBS) and kept at $-80{ }^{\circ} \mathrm{C}$ until use. Before inoculation, the frozen stock was thawed, diluted, and sonicated to disperse clumps.

\subsection{Mice Infection}

The experimental model of pulmonary tuberculosis used in this study has been previously described $[8,9,11]$. Briefly, pathogen-free male BALB/c mice of $6-8$ weeks of age were anesthetized with sevoflurane (Sevorane ${ }^{\circledR}$ ) (Abbvie, IL, EUA) and inoculated intratracheally using a feeding needle with $2.5 \times 10^{5} \mathrm{CFU}$ of the H37Rv Mtb strain.

Twenty-one days post-infection, mice were euthanized, and both lungs were extracted aseptically, snap-frozen in liquid nitrogen, and kept at $-80^{\circ} \mathrm{C}$ until use. All the infection and lung extraction procedures were performed in a Class 3 biological safety cabinet.

\subsection{M. tuberculosis Transcriptome}

To analyze the gene expression for the pathogen and the host, we used both lungs of each mouse to obtain the bacteria and mouse RNA. We performed three strategies to enrich the mycobacterial RNA in the infected lungs and obtain the Mtb transcriptome. Two strategies were implemented during the RNA extraction, and the third, after the sequencing library construction.

\subsection{M. tuberculosis Transcriptome}

RNA extraction. The infected left side lungs were assigned randomly to each strategy, one lung to Strategy 1, and three lungs to Strategies 2 and 3.

(i) RNA extracted without differential lysis and centrifugation (Strategy 1): We pulverized one infected left lung using sterile mortars and pestles frozen with liquid nitrogen and placed the homogenate in a microfuge tube. Then, total RNA was extracted directly using the Quick RNA miniprep Kit (Zymo Research, Irvine, CA, USA; Cat.R1055) following the manufacturer's recommendations, including the digestion with DNAseI to remove the contaminating DNA. Finally, we quantified and assessed the quality of the resulting RNA by Qubit Fluorometer (Invitrogen, Waltham, MA, USA, Cat. Q32851) and Agilent 2100 Bioanalyzer (Agilent Technologies, Santa Clara, CA, USA, Cat. 5067-4626), respectively.

(ii) RNA extracted with differential lysis and centrifugation (Strategy 2): We pulverized six infected left lungs independently using sterile mortars and pestles frozen with liquid nitrogen and placed each homogenate in microfuge tubes. Then, we added RLT buffer (Qiagen, Hilden, Germany, Cat.79216) with $\beta$ mercaptoethanol as the initial lysis buffer to each tube and centrifuged at $14,000 \mathrm{rpm} / 4^{\circ} \mathrm{C}$ for $5 \mathrm{~min}$. We discarded the supernatant and kept the cream-colored pellet on ice. This procedure allowed the enrichment of bacterial cells in the pellet due to the mild-lysis produced by the RLT, which breaks most mouse cells keeping the bacterial cells intact due to its thicker wall membrane. Immediately after centrifugation, we continued the RNA extraction from the pellet using the Quick RNA miniprep Kit (Zymo Research, Irvine, CA, USA; Cat.R1055) following the manufacturer's recommendations, including the digestion with DNAseI to remove the contaminating DNA. Finally, we quantified and assessed the quality of the resulting RNA by Qubit Fluorometer and Agilent 2100 Bioanalyzer, respectively.

Construction of sequencing libraries. The resulting RNA extracted from each lung with Strategies 1 and 2 was treated independently with the Ribo-Zero rRNA Epidemiology Removal Kit (Illumina, San Diego, CA, USA; Cat.MRZE706) following the manufacturers' recommendations. Next, $700 \mu \mathrm{g}$ of depleted RNA was used as input for the NEBNext Ultra RNA Library Prep Kit for Illumina (New England BioLabs, Ipswich, MA, USA; Cat.E7530S), adjusting the Size Select conditions for insert sizes between 400 and $600 \mathrm{bp}$ and the enrichment PCR to 15 cycles. The quantity and quality of all libraries were assessed by Qubit Fluorometer and Agilent 2100 bioanalyzer, respectively. Finally, the library from Strategy 1 and three libraries from strategy 2 were sequenced directly in a NextSeq $500 \mathrm{Mid}$ Output cell in a 150-cycle paired-end format at the National Institute of Genomic Medicine 
(INMEGEN) in Mexico City, Mexico. The remaining three libraries from Strategy 2 were further treated with the in-house ribosomal subtractive hybridization.

In House Ribosomal Substractive hybridization (Strategy 3). Three independent libraries constructed with RNA from Strategy 2 were hybridized with in-house ribosomal probes to reduce Mtb rRNA sequences. Briefly, we amplified the rRNA from the $\mathrm{Mtb}$ genome and sheared the amplicons on a Covaris instrument to an average size of 100-300 bp. The resulting fragments were processed with the NEBNext Fast DNA Library Prep Set for Ion Torrent (New England BioLabs, Ipswich, MA, USA; Cat.E6270S), further amplified with biotinylated primers and purified with AMPure XP beads (Beckman-Coulter, Pasadena, CA, USA; Cat.A63880).

Next, each library was hybridized with the in-house ribosomal probes for $72 \mathrm{hrs}$ using a temperature ramp from $95^{\circ} \mathrm{C}$ to $65^{\circ} \mathrm{C}$. After hybridization, we used magnetic streptavidin-coated beads (Dyabeads MyOne Streptavidin C1; Invitrogen, Waltham, CA, USA; Cat.65001) and washing buffers at a range temperature of $65-99^{\circ} \mathrm{C}$ to gradually pull down the biotinylated probes and separate the captured rRNA. Finally, the non-captured fraction of the libraries was quantified by Qubit Fluorometer, and the quality was assessed by Agilent 2100 bioanalyzer. The non-captured fraction of the libraries were sequenced in a NextSeq 500 Mid Output cell in a 150-cycle paired-end format at the INMEGEN in Mexico City, Mexico.

\subsection{Mouse Transcriptome}

For the host transcriptome, we used the right lung of the same three mice used in Strategy 3 for the Mtb transcriptome.

RNA extraction. We pulverized each of the three lungs using a sterile mortar and pestle frozen with liquid nitrogen. Following the manufacturer's recommendations, the resulting homogenate was processed with the Quick RNA miniprep kit (Zymo Research, Irvine, CA, USA; Cat.R1055), including digestion with DNAseI to remove the contaminating DNA. After the extraction, we quantified and assessed the RNA quality by Qubit Fluorometer and Agilent 2100 bioanalyzer, respectively.

Construction of RNA sequencing libraries.Total RNA from each lung was treated with the NEBNext Poly(A) mRNA Magnetic Isolation Module (New England BioLabs, Ipswich, MA, USA; Cat.E7490) following the manufacturer's recommendations. Then, $700 \mu \mathrm{g}$ of the isolated mRNA was used as input for the NEBNext Ultra RNA Library Prep Kit for Illumina (New England BioLabs, Ipswich, MA, USA; Cat.E7530S), adjusting the Size Select conditions for insert sizes between 400 and $600 \mathrm{bp}$ and the enrichment PCR to 15 cycles. After the procedure, we quantified and assessed each library's quality by Qubit Fluorometer and Agilent 2100 bioanalyzer, respectively. The final libraries were sequenced in a NextSeq500 Mid Output cell in a 300-cycle paired-end format at the INMEGEN in Mexico City, Mexico.

\subsection{Sequencing and Assembly of the M. tuberculosis Infectious Strain}

We extracted the genomic DNA from a previously harvested aliquot of the infecting H37Rv Mtb strain using the Quick-DNA Fecal/Soil Microbe Miniprep Kit (Zymo Research, Irvine, CA, USA; Cat.D6010) following the manufacturer's recommendations. The quantity and quality of the resulting DNA were determined using agarose gel electrophoresis and Qubit fluorometer, respectively.

The sequencing library was constructed with the Nextera XT DNA Library Preparation Kit (Illumina, San Diego, CA, USA; Cat.FC-131-1024) following the manufacturer's recommendations and selecting an insert size of $400-600 \mathrm{bp}$. The final library was quantified with the Qubit fluorometer, and the size distribution was analyzed with Agilent 2100 bioanalyzer. This library was sequenced with the MiSeq Output cell in a 500 cycle paired-end format at the INMEGEN in Mexico City, Mexico.

The sequencing run produced $14,421,346$ total paired reads, $98.1 \%$ of which remained after quality filters $(\geq \mathrm{Q} 20)$ and adaptor removal with FASTX-toolkit (v0.0.13) 
(http:/ / hannonlab.cshl.edu/fastx_toolkit/index.html, accessed on 28 June 2021), and Trimmomatic (v0.36) (http:/ / www.usadellab.org/cms/index.php?page=trimmomatic released 2014, accessed on 28 June 2021). Then, we used SPADES (v.3.13.9) (https://github.com/ablab/spades, accessed on 28 June 2021) and MeDuSa (http:/ / combo.dbe.unifi.it/medusa, accessed on 28 June 2021) to build the de novo assembly. This resulted in 1 contig with 4,392,486 bp and average sequence depth coverage of $788 \times($ Figure $1 \mathrm{~b})$.

\section{a}
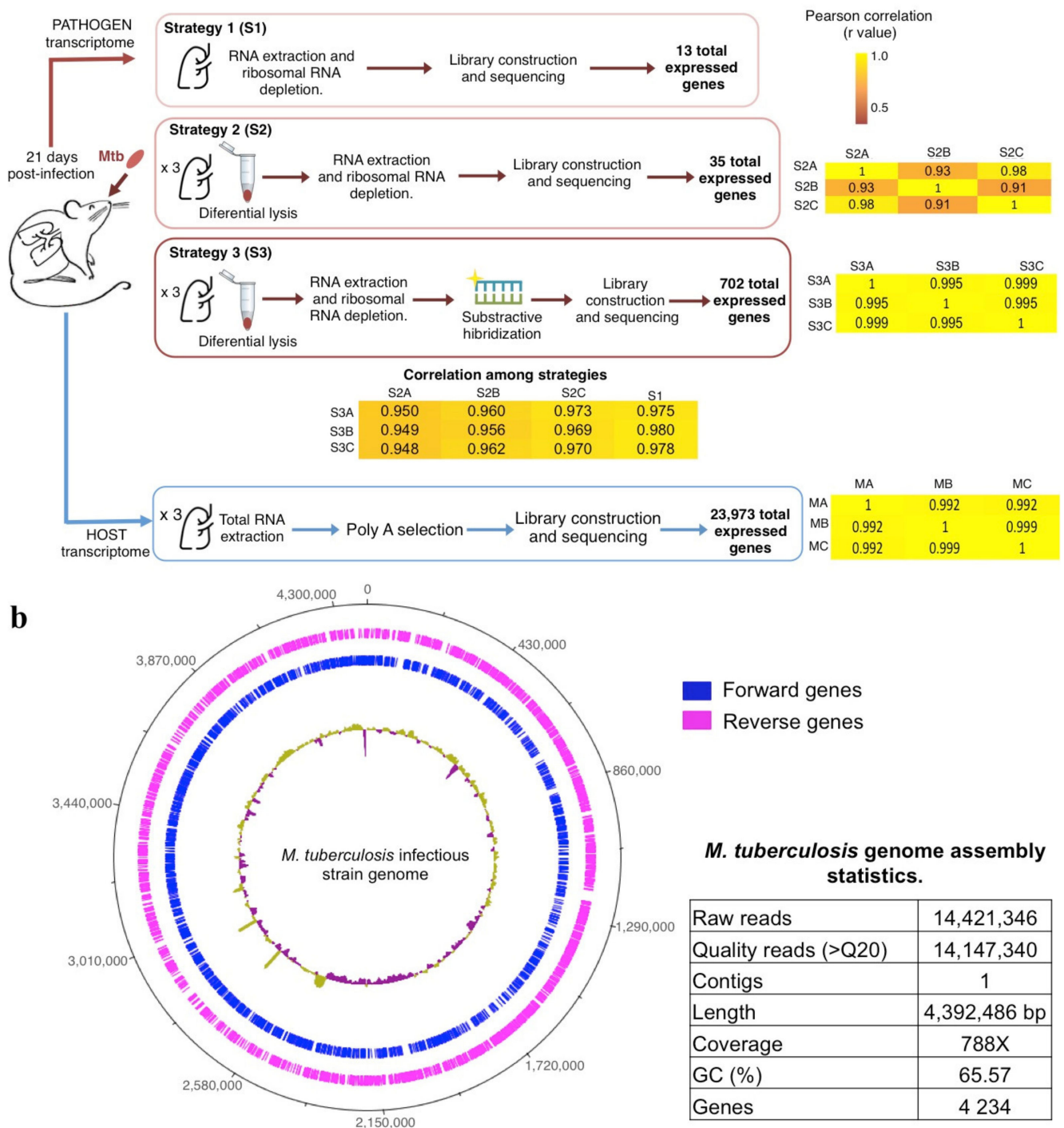

Figure 1. (a) Experimental strategies to obtain the $\mathrm{Mtb}(\mathrm{S} 1, \mathrm{~S} 2$, and $\mathrm{S} 3$ ) and mouse (M) transcriptome profiles. Pearson correlations between three biological replicates (A, B, and C) and strategies (S) are shown in yellow heat maps. (b) Assembled genome of the M. tuberculosis infectious strain. Regions with depth coverage lower and above average $(788 \times)$ are shown as purple and yellow inner circles, respectively. In addition, the statistic parameters of the assembly were reported in the table.

We used Glimmer (v3.02) [12] within Blast2Go (v5.2) [13] with the Prokaryotic Gene Finding feature to locate 4234 genes and determined a homolog for $99.24 \%$ of them analyzing against the non-redundant (nr) database with BLASTX, setting the E-value cut-off at $1.0 \times 10^{-3}$. The transcripts identified as members of the PPE-PGRS family by blast were further searched in the Mycobrowser database [14] to have a more specific description of the PPE-PGRS name. Additionally, all genes were associated with protein families through InterProScan, and functionally mapped to GO terms setting the following parameters: 
E-value-hi-filer: $1.0 \times 10^{-3}$; annotation cut-off: 55 and GO weight: 5 . Finally, the noncoding RNA regions present in the assembled Mtb genome were determined with Infernal (v1.1.3) [15] comparing against the Rfam 14.1 database. The resulting coordinates of the identified regions were added to the gff file with all the annotations.

\subsection{Analysis of Host and Pathogen RNA-Seq Data}

2.7.1. Analysis of M. tuberculosis Transcriptome

Pair-end reads were checked for quality $>20 \mathrm{Q}$ with FASTX-toolkit (v0.0.13), (http:/ / hannonlab.cshl.edu/fastx_toolkit/index.html, accessed on 28 June 2021) and adaptors were trimmed using Trimmomatic (v0.36) (http:/ / www.usadellab.org/cms/index. php?page=trimmomatic, accessed on 28 June 2021). Next, as Avraham et al. 2016 [1] suggested, we built a composed database to analyze the mixed host-pathogen reads and minimize spurious read alignments. This database contained the mouse and Mtb rRNA sequences, mouse reference genome GRCm38.p6 (GenBank GCA_000001635.8), and the de novo assembly of the Mtb infectious genome. All read mappings were performed using SMALT (v0.7.6) (https:/ / github.com/rcallahan/smalt, accessed on 28 June 2021), adjusting strict parameters to avoid cross-mapping reads that will affect transcript quantification. We only considered as positive reads that mapped with a minimum coverage of $\geq 80 \%$.

The reads mapped to rRNA and mouse sequences were counted and separated using the Samtools suite (v1.3.1) (https:/ / sourceforge.net/projects/samtools/files/samtools/, accessed on 28 June 2021). Finally, to determine the number of reads mapped to Mtb genes and non-coding regions, we intersected the aligned bam file with the corresponding gff file containing all the annotations using Bedtools (v2.26.0) (https://github.com/ arq5x/bedtools2/releases, accessed on 28 June 2021). The gene count of each library was normalized by reads per kilobase per million mapped reads (RPKM), and Pearson rank correlations between replicates and between control and experimental libraries were calculated with the GraphPad Prism7 (GraphPad software, San Diego, CA, USA).

Further, we used Blast2Go (BioBam Bioinformatics, Valencia, Spain) to perform an InterPro and gene ontology (GO) enrichment analysis using Fisher's exact test, considering a significant $p$-value of $<0.05$ and the complete $\mathrm{Mtb}$ assembled genome as a reference. We used the KEGG database with a bi-directional best-hit method (BBH) and Mycobacterium tuberculosis as the reference gene set, then determined the percentage of each pathway represented by our set of expressed genes.

To analyze the presence of expressed genes corresponding to secreted proteins, we aligned the sequences of the 529 most expressed genes against the published secretome of $M$. tuberculosis [16], considering as positive an E-value $<0.001$ and coverage $>70 \%$. Additionally, we constructed 100 groups of 529 genes randomly selected from the 4234 total $\mathrm{Mtb}$ genes. Then, we compared each group with the Mtb secretome and determined the number of groups resulting in more than 85 proteins aligned with an E-value $<0.001$ and $>70 \%$ coverage.

Finally, to analyze if the distribution of the 12 genes from the Top 20 list in the $1138 \mathrm{~kb}$ region was a random event, we first divided our Mtb assembled genome into four sections, each accounting for $\sim 25 \%$ of the total length. Next, we created a hundred groups of 20 random genes selected from the 702 observed expressed genes and observed their location. Lastly, for each group, we counted the number of genes located within the same region of the genome and considered a positive event if there were 12 or more genes located together.

\subsubsection{Analysis of Mouse Transcriptome}

Pair-end reads were checked for quality $>20$ Q with FASTX-toolkit (v0.0.13) (http:/ / hannonlab.cshl.edu/fastx_toolkit/index.html, accessed on 28 June 2021), and adaptors were trimmed using Trim_Galore (v0.4.2) (https:/ / github.com/FelixKrueger/TrimGalore, accessed on 28 June 2021). Next, filtered reads were aligned against the Mus musculus NCBI reference genome GRCm38.p6 (GenBank GCA_000001635.8) using Bowtie2 (v2.3.5) (http://bowtie-bio.sourceforge.net/bowtie2/index.shtml, accessed on 28 June 2021) with 
default parameters. Next, the raw count expression profiles were obtained using HTSeq (version 0.6.1) (https:/ /htseq.readthedocs.io/en/master/history.html\#version-0-6-1, accessed on 28 June 2021), then, gene count was normalized by RPKM.

We used the gene ontology (GO) database to analyze enriched GO terms in the Biological Process, Molecular Function, and Cellular Component categories using a Fisher's exact test, a significant $p$-Value of $<0.05$, and Mus musculus as the reference genome. Additionally, we used the KEGG database to analyze the most abundant KEGG pathways with a bi-directional best-hit method (BBH) and Mus musculus as the reference gene set.

\section{Results and Discussion}

\subsection{The Differential Cell Lysis Protocol Concentrated the Number of Mycobacterial Cells and} Increased the Extracted Bacterial RNA

As a first approach, we sequenced the total RNA extracted from one infected lung (Figure 1a) to explore the expression obtained following the typical RNA-seq strategy (Strategy 1, S1). Next, we analyzed the sequencing data mapping against a multifasta file containing the mouse reference genome, Mtb rRNA sequences, and the Mtb strain genome used for the infection (see materials and methods) (Figure 1b). The analysis showed that only $1.70 \%$ of sequences belonged to $\mathrm{Mtb}$, representing 13 expressed genes (Table S1).

Thus, we standardized a differential cell lysis protocol to concentrate the number of mycobacterial cells before the RNA extraction (Strategy 2, S2) (Figure 1a). In this case, the Zihel-Neelsen staining showed a bacterial cell concentration (Figure 2), and the sequences from three biological replicates confirmed a $\sim 4 \%$ increase in the proportion of Mtb sequences compared to S1 (Table S1).
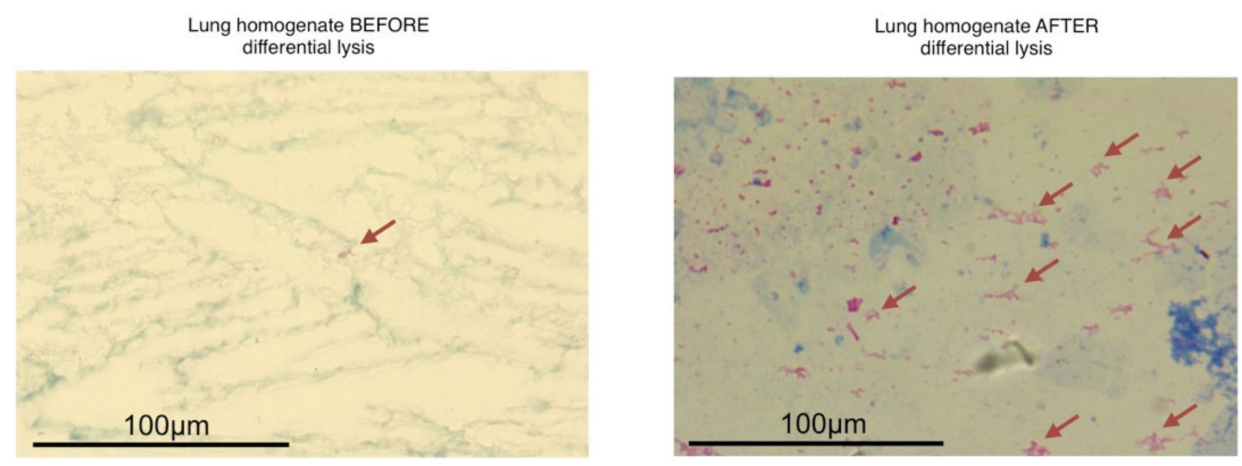

Figure 2. Zihel-Neelsen staining of the infected lung homogenate before and after differential cellular lysis. The red arrow shows the bacilli per field.

However, despite using a ribosomal depletion kit, $97 \%$ of the Mtb sequences still belonged to ribosomal transcripts, and the remaining sequences represented the expression of 35 genes. Some studies have reported that commercial kits remove $70-85 \%$ of ribosomal RNA in pure mycobacterial cultures [17]. However, our samples derived from infected tissue, and the mix of host and mycobacteria cells might have reduced the depletion efficiency.

To reduce the amount of ribosomal RNA, we designed biotinylated probes to selectively subtract the ribosomal transcripts from the total RNA of three independent infected lungs (Strategy 3, S3) (Figures 1a and 3a) (see materials and methods). This method successfully decreased $24.56 \%$ of Mtb ribosomal transcripts, allowing the observation of 702 expressed genes (Table S2), a 50 fold enrichment in the gene number compared to S1. Additionally, Pearson correlations of the gene expression abundance between strategies showed no bias due to the differential lysis and probe hybridization methods $(r>0.9)$ as well as good reproducibility among samples (Figure 1a). 


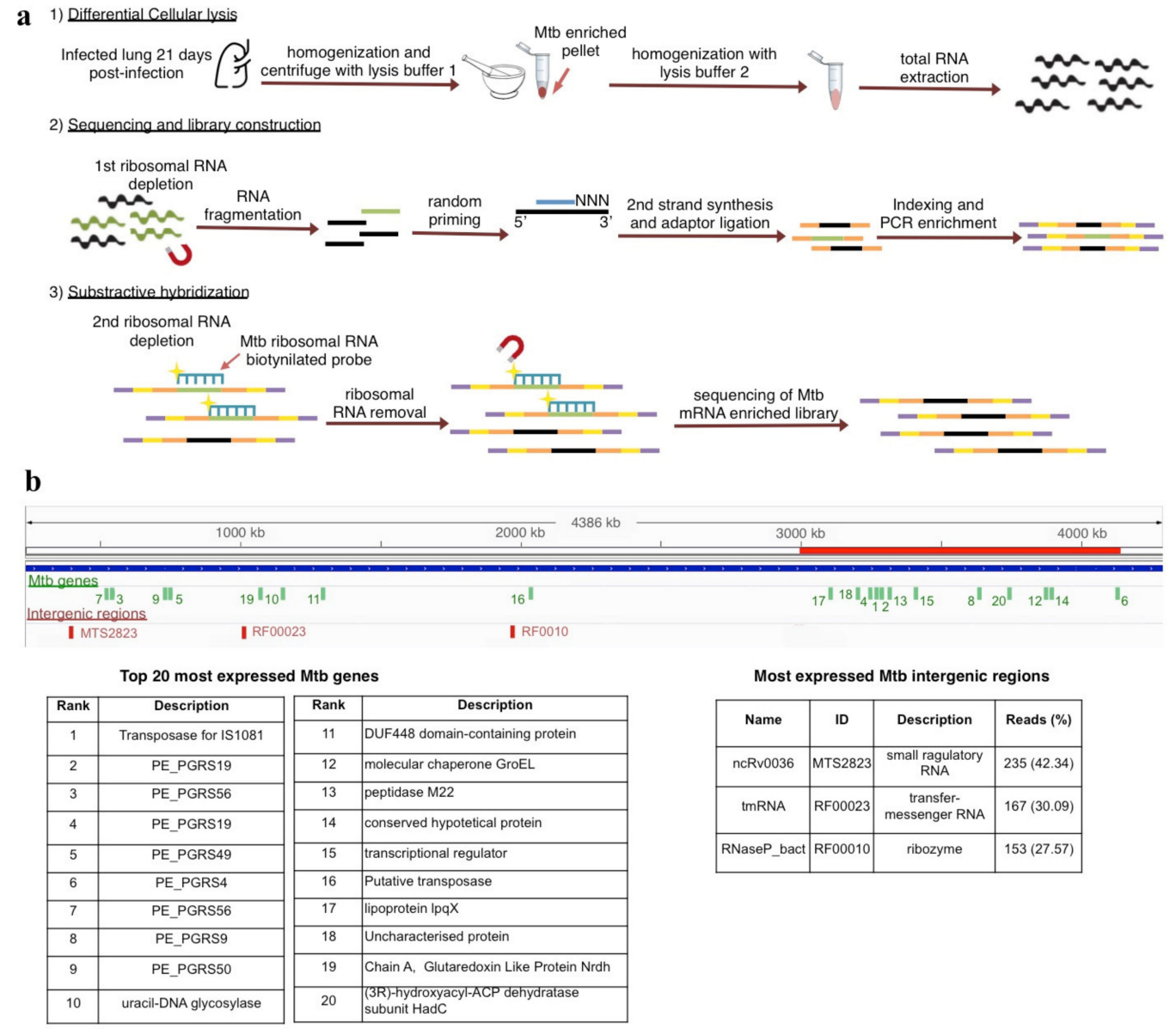

Figure 3. Final strategy to obtain Mtb gene expression profile and genome location of the twenty most expressed genes. (a) Detailed experimental procedure used to obtain the transcriptome profile in Strategy 3. (b) Genomic location of the Top 20 most expressed Mtb genes (green vertical lines) and the three most expressed intergenic regions (red vertical lines) across the Mtb-sequenced genome. The red horizontal line indicates the $1138 \mathrm{~kb}$ region of the Mtb genome (from 3,005,716 bp to $4,144,624 \mathrm{bp}$ ) that concentrated twelve of the top 20 most expressed genes. The tables indicate each expressed gene ranked from the most to the least expressed and the percentage of reads mapped to each intergenic region.

\subsection{The MTb Gene Expression Profile Showed a High Abundance of Genes Codifiyng PE-PGRS Members and the Insertion Sequence IS1081}

We used the data obtained with Strategy 3 (S3) to describe the gene expression profile of Mtb during the in vivo infection. Interestingly, the data showed that $61.59 \%$ of the non-ribosomal Mtb sequences represented 702 genes (Table S2), while $38.41 \%$ represented 30 intergenic regions. To avoid analyzing transitory transcripts, we only considered 529 genes with $\geq 2$ reads in at least one sample and an average RPKM $\geq 1$ for further analysis (Table S2).

First, we determined the InterPro and Gene Ontology (GO) annotations significantly $(p<0.05)$ enriched in the 529 most expressed genes as compared to the complete Mtb genome. The InterPro analysis showed a significant $(p<0.05)$ enrichment of eight protein domains related to fatty acid metabolism and two protein families representing type VII secretion system subunits (Table 1 and Table S3). 
Table 1. InterPro domains and families significantly overrepresented in the 529 most expressed M. tuberculosis genes.

\begin{tabular}{cccc}
\hline InterPro ID & Category & Description & $p$-Value \\
\hline IPR014043 & domain & Acyl transferase & $6.267 \times 10^{-7}$ \\
IPR009081 & domain & Phosphopantetheine binding ACP domain & $1.438 \times 10^{-6}$ \\
IPR014030 & domain & Beta-ketoacyl synthase, N-terminal & $2.747 \times 10^{-5}$ \\
IPR014031 & domain & Beta-ketoacyl synthase, C-terminal & $4.885 \times 10^{-5}$ \\
IPR013968 & domain & Polyketide synthase, ketoreductase domain & $1.624 \times 10^{-4}$ \\
IPR023836 & family & EccCa-like, Actinobacteria & $1.087 \times 10^{-3}$ \\
IPR023837 & family & EccCb-like, Actinobacteria & $1.087 \times 10^{-3}$ \\
IPR032821 & domain & Ketoacyl-synthetase, C-terminal extension & $1.190 \times 10^{-3}$ \\
IPR006091 & domain & Acyl-CoA oxidase/dehydrogenase, central & $1.363 \times 10^{-3}$ \\
IPR020807 & domain & Polyketide synthase, dehydratase domain & $1.757 \times 10^{-3}$ \\
IPR009075 & domain & Acyl-CoA dehydrogenase/oxidase C-terminal & $1.995 \times 10^{-3}$ \\
IPR002543 & domain & FtsK domain & $6.180 \times 10^{-3}$ \\
IPR003029 & domain & S1 domain & $7.039 \times 10^{-3}$ \\
IPR003495 & domain & CobW/HypB/UreG, nucleotide-binding & $7.039 \times 10^{-3}$ \\
IPR023753 & domain & domain & $1.360 \times 10^{-2}$ \\
IPR000788 & domain & Ribonucleotide reductase large subunit, & $1.558 \times 10^{-2}$ \\
IPR001030 & domain & C-terminal & \\
IPR002300 & domain & Aconitase/3-isopropylmalate dehydratase large & $1.558 \times 10^{-2}$ \\
IPR003714 & domain & Aminoacyl-tRNA synthetase, class Ia & $1.558 \times 10^{-2}$ \\
IPR004100 & domain & ATPase, F1/V1/A1 complex, alpha/beta & $1.558 \times 10^{-2}$ \\
& & subunit, N-terminal domain & $1.558 \times 10^{-2}$ \\
\hline
\end{tabular}

As expected, the GO analysis showed a significant $(p<0.05)$ enrichment of terms related to lymphocyte and T-cell co-stimulation, T-cell receptors, nitrate reductase, zymogen binding, and scavenger response (Table 2 and Table S4). Accordingly, the KEGG pathway analyses of the 529 most expressed genes showed fatty acid metabolism, secretion, Tcell and lymphocyte co-stimulation, glyoxylate and dicarboxylate metabolism, fatty acid synthesis, secretion systems, and quorum sensing as some of the pathways covered $\geq 20 \%$ by the expressed genes (Table 3 and Table S5).

The InterPro, GO, and KEGG analysis showed an enrichment of terms related to energy production and macromolecules synthesis. This profile was expected and is consistent with the exponential growth phase of the bacteria during this infection stage [8,9]. Moreover, the abundant presence of pathways for glyoxylate metabolism indicated that the mycobacteria are adapting to a stressful environment [4] using lipids as a carbon source [18]. Additionally, the expression of genes related to T-cell stimulation and cell recruitment is consistent with the day post-infection we are analyzing (day 21), where the granuloma formation occurs [8,9].

The most over-expressed Mtb gene was the transposase for IS1081 (Rv2512c) (Table 4), an insertion sequence used as a molecular marker for $M$. bovis and Mtb but with no previous reports of expression during Mtb in vivo infections. In contrast, the insertion sequence IS6110, besides being considered the standard molecular marker for Mtb strains [19], has also been proposed as a promoter that can affect mycobacterial fitness given its location within the genome of some strains [19]. Some authors suggest it can affect the bacterial gene expression by causing a frameshift that affects the transcription or producing an RNA pseudoknot, which interferes with translation [20]. Additionally, increased transposase activity for IS6110 was observed in mice infected with Mtb H37Rv and in bacterial liquid cultures with nutrient deficiency [20]. These results suggest that the transposition of IS6110 may respond to stress conditions such as the one produced during an in vivo infection. However, the IS6110 transposase showed a low expression ranking under 300 of the 529 expressed genes in our data. 
Table 2. Gene Ontology terms significantly enriched by category related to the 529 most expressed M. tuberculosis gene separated by category.

\begin{tabular}{|c|c|c|}
\hline GO ID & GO Name & $p$-Value \\
\hline \multicolumn{3}{|c|}{ A. Biological Process } \\
\hline GO:0031295 & T-cell co-stimulation & $1.765 \times 10^{-5}$ \\
\hline GO:0031294 & Lymphocyte co-stimulation & $1.765 \times 10^{-5}$ \\
\hline GO:0009081 & Branched-chain amino acid metabolic process & $4.007 \times 10^{-5}$ \\
\hline GO:0009083 & Branched-chain amino acid catabolic process & $1.102 \times 10^{-4}$ \\
\hline GO:0006549 & Isoleucine metabolic process & $1.197 \times 10^{-4}$ \\
\hline GO:0000288 & $\begin{array}{l}\text { Nuclear-transcribed mRNA catabolic process, } \\
\text { Deadenylation-dependent decay }\end{array}$ & $2.663 \times 10^{-4}$ \\
\hline GO:0006573 & Valine metabolic process & $3.513 \times 10^{-4}$ \\
\hline GO:0060184 & Cell cycle switching & $4.878 \times 10^{-4}$ \\
\hline GO:0051728 & Cell cycle switching, mitotic to meiotic cell cycle & $4.878 \times 10^{-4}$ \\
\hline GO:0006574 & Valine catabolic process & $5.108 \times 10^{-4}$ \\
\hline \multicolumn{3}{|c|}{ B. Cellular Component } \\
\hline GO:0005643 & Nuclear pore & $4.20 \times 10^{-3}$ \\
\hline GO:0000932 & P-body & $5.39 \times 10^{-3}$ \\
\hline GO:0009986 & Cell surface & $6.00 \times 10^{-3}$ \\
\hline GO:0098978 & Glutamatergic synapse & $6.18 \times 10^{-3}$ \\
\hline GO:1990527 & Tec1p-Ste12p-Dig1p complex & $7.04 \times 10^{-3}$ \\
\hline GO:0110165 & Cellular anatomical entity & $1.23 \times 10^{-2}$ \\
\hline GO:1990526 & Ste12p-Dig1p-Dig2p complex & $1.60 \times 10^{-2}$ \\
\hline GO:0030496 & Midbody & $1.81 \times 10^{-2}$ \\
\hline GO:0009325 & Nitrate reductase complex & $1.83 \times 10^{-2}$ \\
\hline GO:0016020 & Membrane & $2.56 \times 10^{-2}$ \\
\hline \multicolumn{3}{|c|}{ C. Molecular Function } \\
\hline GO:0004085 & Butyryl-CoA dehydrogenase activity & $1.62 \times 10^{-4}$ \\
\hline GO:0005488 & Binding & $6.96 \times 10^{-4}$ \\
\hline GO:0052890 & $\begin{array}{l}\text { Oxidoreductase activity, acting on the } \mathrm{CH}-\mathrm{CH} \text { group } \\
\text { of donors, with a flavin as acceptor }\end{array}$ & $7.83 \times 10^{-4}$ \\
\hline GO:0043168 & Anion binding & $1.16 \times 10^{-3}$ \\
\hline GO:0003955 & $\mathrm{NAD}(\mathrm{P}) \mathrm{H}$ dehydrogenase (quinone) activity & $1.94 \times 10^{-3}$ \\
\hline GO:0017056 & Structural constituent of nuclear pore & $2.87 \times 10^{-3}$ \\
\hline GO:0004962 & Endothelin receptor activity & $2.94 \times 10^{-3}$ \\
\hline GO:0036094 & Small molecule binding & $3.15 \times 10^{-3}$ \\
\hline GO:1901363 & Heterocyclic compound binding & $4.09 \times 10^{-3}$ \\
\hline GO:0097159 & Organic cyclic compound binding & $4.10 \times 10^{-3}$ \\
\hline
\end{tabular}

Table 3. KEGG pathways overrepresented by the 529 most expressed M. tuberculosis genes.

\begin{tabular}{ccc}
\hline KEGG Id & Pathway & $\begin{array}{c}\text { \% of the Pathway } \\
\text { Represented by the } \\
\text { Expressed Genes }\end{array}$ \\
\hline 03020 & RNA polymerase & 75.00 \\
00630 & Glyoxylate and dicarboxylate metabolism & 34.15 \\
03018 & RNA degradation & 33.33 \\
00562 & Inositol phosphate metabolism & 33.33 \\
00910 & Nitrogen metabolism & 31.82 \\
05152 & Tuberculosis & 30.77 \\
00680 & Methane metabolism & 28.57 \\
00290 & Valine, leucine and isoleucine biosynthesis & 28.57 \\
00430 & Taurine and hypotaurine metabolism & 28.57 \\
00020 & Citrate cycle (TCA cycle) & 28.13 \\
00730 & Thiamine metabolism & 27.27 \\
00983 & Drug metabolism-other enzymes & 27.27 \\
\hline
\end{tabular}


Table 3. Cont.

\begin{tabular}{ccc}
\hline KEGG Id & Pathway & $\begin{array}{c}\text { \% of the Pathway } \\
\text { Represented by the } \\
\text { Expressed Genes }\end{array}$ \\
\hline 00061 & Fatty acid biosynthesis & 26.67 \\
02024 & Quorum sensing & 25.00 \\
00010 & Glycolysis/Gluconeogenesis & 24.24 \\
00270 & Cysteine and methionine metabolism & 24.24 \\
00260 & Glycine, serine, and threonine metabolism & 24.00 \\
03060 & Protein export & 23.53 \\
01053 & Biosynthesis of siderophore group nonribosomal peptides & 22.22 \\
03070 & Bacterial secretion system & 21.43 \\
\hline
\end{tabular}

Table 4. Top 20 most expressed M. tuberculosis genes at day 21 post-infection.

\begin{tabular}{cccc}
\hline $\begin{array}{c}\text { Expression } \\
\text { Ranking }\end{array}$ & Gen ID & Gene Description & Mean RPKM \\
\hline 1 & Rv2512c & Transposase for insertion sequence element IS1081 & $6.84 \times 10^{5}$ \\
2 & Rv1067c & PE_PGRS19 PE-PGR & $3.45 \times 10^{5}$ \\
3 & Rv3512 & PE_PGRS56 PE-PGRS & $1.91 \times 10^{5}$ \\
4 & Rv1067c & PE_PGRS19 PE-PGR & $1.65 \times 10^{5}$ \\
5 & Rv3344c & PE_PGRS49 PE-PGR & $1.63 \times 10^{5}$ \\
6 & Rv0279c & PE_PGRS4 PE-PGRS & $1.10 \times 10^{5}$ \\
7 & Rv3512 & PE_PGRS56 PE-PGRS & $5.01 \times 10^{4}$ \\
8 & Rv0746 & PE_PGRS9 PE-PGRS & $4.35 \times 10^{4}$ \\
9 & Rv3345c & PE_PGRS50 PE-PGR & $1.94 \times 10^{4}$ \\
10 & Rv0105c & uracil-DNA glycosylase & $7.61 \times 10^{3}$ \\
11 & Rv2840c & DUF448 domain-containing protein & $6.94 \times 10^{3}$ \\
12 & Rv0440 & molecular chaperone GroEL & $5.71 \times 10^{3}$ \\
13 & Rv3620c & peptidase M22 & $4.88 \times 10^{3}$ \\
14 & Rv0454 & conserved hypotetical protein & $4.58 \times 10^{3}$ \\
15 & Rv0967 & transcriptional regulator & $4.12 \times 10^{3}$ \\
16 & Rv2424c & Putative transposase & $4.05 \times 10^{3}$ \\
17 & Rv1228 & lipoprotein lpqX & $3.66 \times 10^{3}$ \\
18 & Rv2424c & Uncharacterized protein & $3.11 \times 10^{3}$ \\
19 & Rv3053c & Chain A, Glutaredoxin Like Protein Nrdh & $3.09 \times 10^{3}$ \\
20 & Rv0637 & (3R)-hydroxyacyl-ACP dehydratase subunit HadC & $2.96 \times 10^{3}$ \\
\hline
\end{tabular}

Notably, we observed that the genome of Mtb H37Rv has 33 copies for the IS6110 transposase while the IS1081 transposase has only 6, suggesting that the high expression of the transposase for IS1081 we observed was not due to a higher gene copy number. Thus, suggesting the importance of considering the role of IS1081 during the development of Mtb in different environments.

The following eight most expressed genes belonged to the PE-PGRS family (Table 4), considered important Mtb antigens that are in close contact with the host immune system [21]. Particularly, PGRS49 and PGRS50 have been proposed as strong vaccine candidates [22] due to their strong antigenicity. On the other hand, PE-PGRS9 and PE-PGRS53 had been previously related to chronic Mtb infections [21,23]. Interestingly, only one previous in vivo study [6] reported the expression of PE-PGRS members, but not as the primarily expressed genes. Thus, to the best of our knowledge, this is the first RNA-seq study to highlight the role of some PE-PGRS transcripts during the early stages of tuberculosis in vivo infection. In line with the high expression of PE-PGRS, we also observed an increased expression of secretion system subunits necessary to export them, especially ESX-1, ESX-3, and ESX-5 [24].

Interestingly, 12 of the 20 most expressed genes were located within a $1138 \mathrm{~kb}$ region, representing $25.95 \%$ of the genome (Figure $3 \mathrm{~b}$ and Table S2). To test if this observation was 
coincidental, we formed 100 groups of 20 random genes selected from the complete set of 702 expressed genes observed and determined their location. Notably, neither group showed more than nine genes within the same region $(p=0.00)$.

In a previous study, Talaat [6] defined a $34.1 \mathrm{~kb}$ region concentrating 20 significantly expressed genes during in vivo infection, and interestingly, our $1138 \mathrm{~kb}$ region contained five of those 20 genes. These results suggest that the transcription of this section of the genome is essential for the host-pathogen interaction.

\subsection{An Increased Proportion of Expressed Genes Belong to the MTb Secretome}

We compared how many of the 529 expressed genes corresponded to the Mtb secretome previously reported [16]. According to the previous publication [16], we expected that only $\sim 12 \%$ (63 genes) belonged to the secretome, but we observed that $16.07 \%$ ( 85 genes) codified for secreted proteins (Table S6). These suggested a slight overabundance of the genes for secreted proteins in the transcriptome. To test if this observation was coincidental, we formed 100 groups of 529 random genes selected from 4234 total Mtb genes and compared their presence in the secretome. As a result, 41 from the 100 groups had more than 85 genes aligned with the secretome (E-value $<0.001$ and $>70 \%$ coverage). These results suggest that the over-abundance of genes for secreted proteins had a low probability of being a random event.

Among the expressed transcripts for secreted proteins, we observed seven lipoproteins, such as lppN (Rv2270) and LpqH (Rv3763), which are considered important for the hostpathogen interaction (Table S6). Some studies suggest they help internalize the bacteria into the macrophages [25]. Particularly LpqH has shown some effects that favor the host, such as increasing IL-12, while other effects favor the pathogen, such as inhibiting INF $\gamma$, and decreasing the expression of MCH-II and antigen processing [26-28]. In the study by Pisu [5], they detect some lipoproteins responsible for the degradation of triglycerides and cholesterol overexpressed in infected alveolar macrophages, such as Lpl and LipA. However, they did not detect overexpression of $\mathrm{LpqH}$ or lppN, probably because these proteins respond to different stimuli in other cell types.

On the other hand, $28.24 \%$ (24 genes) of the 85 expressed transcripts for secreted proteins were members of the PE-PGRS family (Table S6). Four of them were some of the most expressed genes (PGRS-49, PGRS56, PGRS9 y PGRS50) (Table 4). We also observed PE68, a gene codified in the region of difference 1 (RD1), which is absent in non-virulent Mtb strains such as BCG [29]. PE68 is considered an efficient immunomodulator that stimulates the host immune cellular response, even promoting tissue damage. Interestingly, although PE68 is not considered essential for the survival of Mtb, some studies suggest that alterations in its sequence affect strain virulence [29]. This transcript ranked in the 25th place of the 529 expressed genes.

\subsection{A High Expression of Three ncRNAs during Mtb In Vivo Infection}

From the whole Mtb sequences, $38.41 \%$ mapped to 30 intergenic regions. Previous reports showed that the expression of intergenic regions in liquid cultures during exponential growth is around $28 \%$, while during the stationary phase, their expression increased to $58 \%$ [30]. These results suggest that the expression of intergenic regions varies during the development of Mtb in different environments.

Among the intergenic regions, we identified three ncRNAs, namely small regulatory RNA MTS2823 (ncRv0036) (42.34\% of sequences), the transfer-messenger RNA RF00023 $(30.09 \%)$, and the ribozyme RF0010 (27.57\%) (Figure 3b).

There is scarce information about the role of ncRNAs during the Mtb infection. Mainly, MTS2823 is considered necessary for Mtb growth under different environments, and its expression is especially accumulated in stationary phase cultures [30], mouse lungs with chronic infections [30], and even in dormant mycobacteria [31]. Furthermore, previous reports suggest that MTS2823 may mediate the down-regulation of genes expressed in the exponential growth phase [30]. Although the mechanisms remain to be clarified, more 
in vivo studies are needed to determine if MTS2823 down-regulates genes during different stages of infection.

Our results suggest that MTS2823 also plays an essential role during the early stages of in vivo infection. Probably, this expression increases in later stages, favoring the maintenance of the chronic infection. Thus, the exciting idea is to favor its expression earlier to control the spread of infection. Contrary, sRNAs induced in the stationary phase such as MRS0997 and MTS1338 were not detected in our dataset [30].

\subsection{The Host Gene Expression Showed an Inflammatory Profile Consistent with the Early Stage of Infection}

Finally, we sequenced the host RNA from the same infected mice, and after assessing good biological reproducibility (Pearson $r=0.9946$ ) (Figure 1a), we selected 15,677 genes with $\geq 10$ reads in two samples for further analysis (Table S7).

The GO analysis considering the top 5\% most expressed genes (Table S8) showed inflammation, antigen processing and presentation, cellular response to lipopolysaccharides, and bacterial evasion activities as some of the most significantly $(p<0.05)$ enriched terms. Accordingly, the KEGG pathway analysis showed antigen processing and presentation, phagolysosome, IL-17, and TNF- $\alpha$ signaling, and Toll receptors activities as some of the pathways represented in $\geq 10 \%$ (Table S9).

In the top 20 most expressed genes, we observed transcripts for two surfactantassociated proteins (Sftpc and Sftpa), three histocompatibility genes, and microRNA 638. Notably, Sftpa is known to act as an opsonin to enhance the ingestion of Mtb and other pulmonary pathogens by alveolar macrophages [32-34]. However, interestingly, this internalization pathway has also been shown to decrease reactive nitrogen intermediates levels, suggesting binding of Sftpa may be one mechanism by which Mtb reduced the macrophage cytotoxic response [34,35].

The dominant immune cellular response in the early infection has been previously described for this murine model (8), consistent with previous studies [36]. In contrast, the expression of genes related to T-cell and lymphocyte co-stimulation and antigen processing is typically associated with chronic infections [37] but maybe involved with granuloma formation and maintenance in early stages [38].

Interestingly, we observed microRNA mi6381 as one of the twenty most expressed genes. This microRNA has no previous reports associating it to tuberculosis or any other pulmonary disease. On the other hand, several in vitro studies suggest that micro RNAs such as mir155, mir135b, or mir146a play essential roles in mycobacterial infections [39-43]. Mainly mir155 was found overexpressed in mononuclear cells of tuberculosis patients than of healthy individuals [44]. However, we did not observe this miRNA in our list of expressed genes, suggesting that different miRNAs may be active in different infected tissues.

\subsection{Comparisson of Mtb Gene Expression Using Other RNA-Seq Methods during In Vivo Infections}

As mentioned before, to the best of our knowledge, two studies aim to describe the Mtb gene expression during an in vivo infection. Both studies have essential methodological similarities and differences with the present study, which we highlight in Table 5. Thus, it is essential to note that discrepancies in the observed expressed genes among studies could arise from the methodological and biological differences such as the murine model, the mycobacterial strain, or the criteria established by each group to consider genes as expressed for the analysis. In this sense, the study by Pisu et al. can be considered the most related to our work; however, it carries critical methodological differences (Table 5), which make the gene expression results not directly comparable between methods. Despite that, we compared our results with Pisu [5], who used RNA-seq to establish an "in vivo signature" of 180 genes expressed in macrophages, and Talaat [6], who used microarrays to determined 159 genes significantly expressed in in vivo conditions. We observed that only 16 and 17 of our expressed genes were observed in Pisu's and Talaat's studies, respectively (Figure 4), while five genes were shared by Talaat vs. Pisu. Thus, this suggested that 
the observed Mtb gene expression among the three studies depends on the different experimental methods and biological variants, such as the murine model and the bacterial strain used.

Table 5. Main characteristics of previous studies describing M. tuberculosis in vivo gene expression.

\begin{tabular}{|c|c|c|c|}
\hline & Talaat et al., 2004 [6] & Pisu et al., 2020 [5] & $\begin{array}{l}\text { Cornejo-Granados et al., } \\
2021\end{array}$ \\
\hline Mtb strain & $\mathrm{H} 37 \mathrm{Rv}$ & Erdman ATCC 35801 mCherry & $\mathrm{H} 37 \mathrm{Rv}$ \\
\hline Day post-infection analyzed & $7,14,21$ and 28 & 14 & 21 \\
\hline Murine model used & $\begin{array}{c}\mathrm{BALB} / \mathrm{c} \text { and BALB } / \mathrm{c} \\
\text { SCID/SCID }\end{array}$ & C57BL/6J WT & $\mathrm{BALB} / \mathrm{c}$ \\
\hline Route of infection & Intranasal & Intranasal & Intratracheal \\
\hline Infected tissue analyzed & Complete infected lungs & $\begin{array}{l}\text { Alveolar and interstitial } \\
\text { macrophages isolated from } \\
\text { infected lungs }\end{array}$ & Complete infected lungs \\
\hline RNA extraction method & $\begin{array}{c}\text { Total RNA extraction with Tri } \\
\text { Reagent (Trizol) from groups } \\
\text { of } 50 \text { mice }\end{array}$ & $\begin{array}{l}\text { Isolated cells were treated } \\
\text { with Trizol and centrifuged to } \\
\text { pellet mycobacterial cells. } \\
\sim 80 \% \text { of the Trizol (containing } \\
\text { host RNA) was removed. } \\
\text { Finally, total RNA was } \\
\text { extracted with fresh Trizol, } \\
\text { and a proportion of the host } \\
\text { RNA was added back. }\end{array}$ & $\begin{array}{l}\text { Used a differential } \\
\text { centrifugation of individual } \\
\text { lungs with a mild-lysis buffer } \\
\text { to pellet mycobacterial cells. } \\
\text { Plus, removing the } \\
\text { supernatant containing host } \\
\text { RNA followed by total RNA } \\
\text { extraction from the } \\
\text { mycobacterial cells with a } \\
\text { commercial kit. }\end{array}$ \\
\hline Ribosomal RNA elimination & - & $\begin{array}{c}\text { Ribo-Zero Gold rRNA } \\
\text { Removal Kit (Epidemiology) }\end{array}$ & $\begin{array}{c}\text { Ribo-Zero Gold rRNA } \\
\text { Removal Kit (Epidemiology) } \\
\text { and In-house Mtb ribosomal } \\
\text { probes }\end{array}$ \\
\hline $\begin{array}{l}\text { Methodology for gene } \\
\text { expression analysis }\end{array}$ & $\begin{array}{l}\text { DNA Microarrays with } \\
\text { oligonucleotides representing } \\
\text { Mtb coding sequences. }\end{array}$ & RNA-seq & RNA-seq \\
\hline Key insights & $\begin{array}{c}\text {-The expression profile of Mtb } \\
\text { in SCID mice is most similar } \\
\text { to the profile when grown in } \\
\text { broth. } \\
\text {-Around } 49 \text { genes were only } \\
\text { expressed in vivo, and } 20 \text { of } \\
\text { these are contiguous in a } \\
\text { delimited area of the Mtb } \\
\text { genome. }\end{array}$ & $\begin{array}{l}\text {-In vivo signature of } 180 \text { genes } \\
\text { upregulated in macrophages. } \\
\text {-Transcriptional signatures } \\
\text { varied with macrophage } \\
\text { ontology. } \\
\text {-Alveolar macrophages } \\
\text { showed a distinct up } \\
\text { regulation of genes for the } \\
\text { acquisition and use of fatty } \\
\text { acids. } \\
\text {-Interstitial macrophages } \\
\text { showed an up regulation of } \\
\text { genes for iron sequestration. }\end{array}$ & $\begin{array}{l}\text {-About } 62.59 \% \text { of } \\
\text { non-ribosomal sequences } \\
\text { represented } 702 \text { genes, while } \\
38.41 \% \text { represented intergenic } \\
\text { regions. } \\
\text {-The transposase for IS1081 } \\
\text { was the most expressed gene. } \\
\text {-Eight genes for PE-PGRS } \\
\text { members are among the most } \\
\text { expressed genes.-Three highly } \\
\text { expressed ncRNAs (MTS2823, } \\
\text { RF00023, and RF00010) }\end{array}$ \\
\hline
\end{tabular}




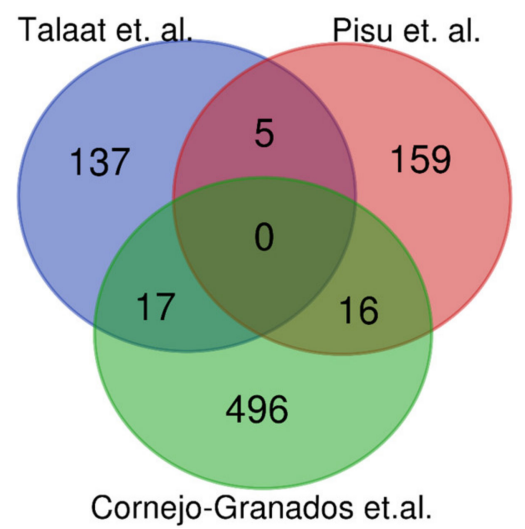

Figure 4. Venn diagram comparing the expressed genes between Talaat et al. [6], Pisu et al. [5], and Cornejo-Granados in vivo studies.

\section{Conclusions}

The experimental method using the differential cell lysis and a probe-based ribosomal depletion presented here allowed the observation of the most expressed genes and intergenic regions of Mtb directly from an in vivo infection, increasing the number of expressed genes observed, from 13 with the traditional approach to 702 using our experimental method.

We acknowledge that the number of genes analyzed could be broadened, increasing the sequencing depth. However, the main objective at this point was to demonstrate the technical efficacy and reproducibility of our experimental method. Additionally, it is important to mention that our analysis only assessed gene expression levels, from which we can only draw limited conclusions. We are conducting studies using this method and using different $\mathrm{Mtb}$ strains, allowing us to understand biological questions using the key value of RNA-seq in a comparative analysis.

The present study revealed the presence of highly expressed MTS2823, a non-coding RNA. Additionally, we observed mi638, a microRNA with no previous reports associating it to tuberculosis or any other pulmonary disease. The analysis we present is a general catalog of the gene expression at one point during the in vivo tuberculosis infection, and it does not intend to draw critical conclusions regarding the pathogen's physiology. In order to better understand the relevance of the expressed genes we observed, it is necessary to analyze and compare additional time points of the disease. In this regard, it is probable that our catalog of expressed genes only reflects the typical cross-talk during the evolution of the host-pathogen interaction. In this sense, our research group has ongoing experiments using this experimental approach to analyze and compare the gene expression during earlier and later stages of the infection and with Mtb strains from different genotypes.

Additionally, it is essential to note that this protocol is not the only experimental approach available for using RNA-seq in an in vivo infection model. As mentioned before, the study by Pisu et al. also uses RNA-seq to analyze the gene expression profile of infected macrophages isolated directly from a murine lung. Definitively, individual cell analysis seams an optimal approach for studying any infectious disease. However, this methodology entails additional expenses in specialized reagents and equipment not typically available for all laboratories. The advantage of our method is that it does not require specialized equipment to conduct a comparative analysis directly from an in vivo infection. Thus, we believe this approach is an efficient and reproducible method that is an alternative for the general scientific community to explore the in vivo gene expression of diverse $\mathrm{Tb}$ strains and clinical isolates with prevalence worldwide.

Supplementary Materials: The following are available online at https: / www.mdpi.com/article/ 10.3390/biology10090848/s1. Table S1: Read count of the three strategies for the M. tuberculosis transcriptome. Table S2: M. tuberculosis genes expressed 21 days post-infection observed with Strategy 3. Table S3: InterPro domains and families significantly overrepresented in the 529 most expressed M. tuberculosis genes. Table S4: Gene Ontology terms significantly enriched by category related to the 
529 most expressed M. tuberculosis gene. Table S5: KEGG pathways overrepresented by the 529 most expressed M. tuberculosis genes. Table S6: M. tuberculosis expressed genes belonging to the predicted secretome. Table S7: 15,677 most expressed mouse genes at day 21 post-infection with $M$. tuberculosis. Table S8: Gene Ontology terms significantly enriched by category related to the most expressed mouse genes. Table S9: KEGG pathways overrepresented by the most expressed mouse genes.

Author Contributions: Conceived and designed the experiments: A.O.-L., R.H.-P. and F.C.-G. Performed the experiments: F.C.-G., D.A.M.-E., J.B.-P., B.M.-C., Z.L.Z.-B. and C.M.-R. Analyzed the data: F.C.-G., G.L.-L. and E.E.-M. Contributed reagents/materials/analysis tools: A.O.-L. and R.H.-P. Wrote the paper: F.C.-G., G.L.-L., D.A.M.-E., J.B.-P., B.M.-C., Z.L.Z.-B., C.M.-R., E.E.-M., R.H.-P. and A.O.-L. All authors have read and agreed to the published version of the manuscript.

Funding: This work was supported by the CONACyT grants SALUD-2014-C01-234188, Salud-201301-202859 and Ciencia de Frontera 2019 263986. This research was also funded by the DGAPA PAPIIT UNAM (IN215520). We also acknowledge the support of program Actividades de Intercambio Académico 2019 CIC-UNAM-CIAD.

Institutional Review Board Statement: All animal procedures were performed according to the national regulations on animal care and experimentation (NOM 062-ZOO-1999) after approval by the Animal Experimentation Committee at the National Institute of Medical Sciences and Nutrition México (CINVA 1329, approved on 23 February 2015). Our study was also carried out in accordance with the ARRIVE (Animal Research: Reporting of In Vivo Experiments) guidelines.

Informed Consent Statement: Not applicable.

Data Availability Statement: The RNA-seq data and the M. tuberculosis genome used in this study have been deposited in NCBI under BioProject number PRJNA669742. All additional results are available in the Supplementary Materials.

Acknowledgments: We thank Juan Manuel Hurtado Ramírez for the informatics technical support and the computational server maintenance. F.C.-G., thanks to the Doctoral Biochemical Sciences Program, at IBT UNAM and CONACyT by doctoral fellowship: C.V.U.: 443238. G.L.-L. acknowledges the support of CONACyT as a postdoctoral fellow. We also thank the Unidad de Secuenciación Masiva from INMEGEN for sequencing technical support.

Conflicts of Interest: The authors declare no conflict of interest.

\section{References}

1. Avraham, R.; Haseley, N.; Fan, A.; Bloom-Ackermann, Z.; Livny, J.; Hung, D.T. A highly multiplexed and sensitive RNA-seq protocol for simultaneous analysis of host and pathogen transcriptomes. Nat. Protoc. 2016, 11, 1477-1491. [CrossRef]

2. Westermann, A.J.; Gorski, S.A.; Vogel, J. Dual RNA-seq of pathogen and host. Nat. Rev. Microbiol. 2012, 10, 618-630. [CrossRef]

3. Schnappinger, D.; Ehrt, S.; Voskuil, M.I.; Liu, Y.; Mangan, J.A.; Monahan, I.M.; Dolganov, G.; Efron, B.; Butcher, P.D.; Nathan, C.; et al. Transcriptional Adaptation of Mycobacterium tuberculosis within Macrophages: Insights into the Phagosomal Environment. J. Exp. Med. 2003, 198, 693-704. [CrossRef]

4. Rohde, K.H.; Abramovitch, R.B.; Russell, D.G. Mycobacterium tuberculosis invasion of macrophages: Linking bacterial gene expression to environmental cues. Cell Host Microbe 2007, 2, 352-364. [CrossRef] [PubMed]

5. Pisu, D.; Huang, L.; Grenier, J.K.; Russell, D.G. Dual RNA-Seq of Mtb-Infected Macrophages In Vivo Reveals Ontologically Distinct Host-Pathogen Interactions. Cell Rep. 2020, 30, 335-350. [CrossRef] [PubMed]

6. Talaat, A.M.; Lyons, R.; Howard, S.T.; Johnston, S.A. The temporal expression profile of Mycobacterium tuberculosis infection in mice. Proc. Natl. Acad. Sci. USA 2004, 101, 4602-4607. [CrossRef] [PubMed]

7. Waddell, S.J.; Laing, K.; Senner, C.; Butcher, P.D. Microarray analysis of defined Mycobacterium tuberculosis populations using RNA amplification strategies. BMC Genom. 2008, 9, 94. [CrossRef] [PubMed]

8. Hernandez-Pando, R.; Orozco, H.; Arriaga, K.; Sampieri, A.; Larriva-Sahd, J.; Madrid-Marina, V. Analysis of the local kinetics and localization of interleukin-1 alpha, tumour necrosis factor-alpha and transforming growth factor-beta, during the course of experimental pulmonary tuberculosis. Immunology 1997, 90, 607-617. [CrossRef] [PubMed]

9. Hernandez-Pando, R.; Orozcoe, H.; Sampieri, A.; Pavon, L.; Velasquillo, C.; Larriva-Sahd, J.; Alcocer, J.M.; Madrid, M.V. Correlation between the kinetics of Th1, Th2 cells and pathology in a murine model of experimental pulmonary tuberculosis. Immunology 1996, 89, 26-33. [PubMed]

10. Bini, E.I.; Mata Espinosa, D.; Marquina Castillo, B.; Barrios Payan, J.; Colucci, D.; Cruz, A.F.; Zatarain, Z.L.; Alfonseca, E.; Pardo, M.R.; Bottasso, O.; et al. The influence of sex steroid hormones in the immunopathology of experimental pulmonary tuberculosis. PLoS ONE 2014, 9, e93831. [CrossRef] [PubMed] 
11. Lopez, B.; Aguilar, D.; Orozco, H.; Burger, M.; Espitia, C.; Ritacco, V.; Barrera, L.; Kremer, K.; Hernandez-Pando, R.; Huygen, K.; et al. A marked difference in pathogenesis and immune response induced by different Mycobacterium tuberculosis genotypes. Clin. Exp. Immunol. 2003, 133, 30-37. [CrossRef] [PubMed]

12. Delcher, A.L.; Harmon, D.; Kasif, S.; White, O.; Salzberg, S.L. Improved microbial gene identification with GLIMMER. Nucleic Acids Res. 1999, 27, 4636-4641. [CrossRef] [PubMed]

13. Conesa, A.; Gotz, S. Blast2GO: A comprehensive suite for functional analysis in plant genomics. Int. J. Plant Genom. 2008, 2008, 619832. [CrossRef] [PubMed]

14. Kapopoulou, A.; Lew, J.M.; Cole, S.T. The MycoBrowser portal: A comprehensive and manually annotated resource for mycobacterial genomes. Tuberculosis 2011, 91, 8-13. [CrossRef]

15. Nawrocki, E.P.; Eddy, S.R. Infernal 1.1: 100-fold faster RNA homology searches. Bioinformatics 2013, 29, 2933-2935. [CrossRef]

16. Cornejo-Granados, F.; Zatarain-Barron, Z.L.; Cantu-Robles, V.A.; Mendoza-Vargas, A.; Molina-Romero, C.; Sanchez, F.; Del Pozo-Yauner, L.; Hernandez-Pando, R.; Ochoa-Leyva, A. Secretome Prediction of Two M. tuberculosis Clinical Isolates Reveals Their High Antigenic Density and Potential Drug Targets. Front. Microbiol. 2017, 8, 128. [CrossRef]

17. Wang, S.; Dong, X.; Zhu, Y.; Wang, C.; Sun, G.; Luo, T.; Tian, W.; Zheng, H.; Gao, Q. Revealing of Mycobacterium marinum transcriptome by RNA-seq. PLoS ONE 2013, 8, e75828. [CrossRef]

18. Russell, D.G.; VanderVen, B.C.; Lee, W.; Abramovitch, R.B.; Kim, M.J.; Homolka, S.; Niemann, S.; Rohde, K.H. Mycobacterium tuberculosis wears what it eats. Cell Host Microbe 2010, 8, 68-76. [CrossRef]

19. Roychowdhury, T.; Mandal, S.; Bhattacharya, A. Analysis of IS6110 insertion sites provide a glimpse into genome evolution of Mycobacterium tuberculosis. Sci. Rep. 2015, 5, 12567. [CrossRef]

20. Gonzalo-Asensio, J.; Perez, I.; Aguilo, N.; Uranga, S.; Pico, A.; Lampreave, C.; Cebollada, A.; Otal, I.; Samper, S.; Martin, C. New insights into the transposition mechanisms of IS6110 and its dynamic distribution between Mycobacterium tuberculosis Complex lineages. PLoS Genet. 2018, 14, e1007282. [CrossRef]

21. Delogu, G.; Sanguinetti, M.; Pusceddu, C.; Bua, A.; Brennan, M.J.; Zanetti, S.; Fadda, G. PE_PGRS proteins are differentially expressed by Mycobacterium tuberculosis in host tissues. Microbes Infect. 2006, 8, 2061-2067. [CrossRef]

22. Bettencourt, P.; Muller, J.; Nicastri, A.; Cantillon, D.; Madhavan, M.; Charles, P.D.; Fotso, C.B.; Wittenberg, R.; Bull, N.; Pinpathomrat, N.; et al. Identification of antigens presented by MHC for vaccines against tuberculosis. NPJ Vaccines 2020, 5, 2. [CrossRef]

23. Kruh, N.A.; Troudt, J.; Izzo, A.; Prenni, J.; Dobos, K.M. Portrait of a pathogen: The Mycobacterium tuberculosis proteome in vivo. PLoS ONE 2010, 5, e13938. [CrossRef] [PubMed]

24. Bitter, W.; Houben, E.N.; Luirink, J.; Appelmelk, B.J. Type VII secretion in mycobacteria: Classification in line with cell envelope structure. Trends Microbiol. 2009, 17, 337-338. [CrossRef] [PubMed]

25. Ocampo, M.; Curtidor, H.; Vanegas, M.; Patarroyo, M.A.; Patarroyo, M.E. Specific interaction between Mycobacterium tuberculosis lipoprotein-derived peptides and target cells inhibits mycobacterial entry in vitro. Chem. Biol. Drug Des. 2014, 84, 626-641. [CrossRef] [PubMed]

26. Stewart, G.R.; Wilkinson, K.A.; Newton, S.M.; Sullivan, S.M.; Neyrolles, O.; Wain, J.R.; Patel, J.; Pool, K.L.; Young, D.B.; Wilkinson, R.J. Effect of deletion or overexpression of the 19-kilodalton lipoprotein Rv3763 on the innate response to Mycobacterium tuberculosis. Infect. Immun. 2005, 73, 6831-6837. [CrossRef]

27. Noss, E.H.; Pai, R.K.; Sellati, T.J.; Radolf, J.D.; Belisle, J.; Golenbock, D.T.; Boom, W.H.; Harding, C.V. Toll-like receptor 2-dependent inhibition of macrophage class II MHC expression and antigen processing by 19-kDa lipoprotein of Mycobacterium tuberculosis. J. Immunol. 2001, 167, 910-918. [CrossRef]

28. Gehring, A.J.; Rojas, R.E.; Canaday, D.H.; Lakey, D.L.; Harding, C.V.; Boom, W.H. The Mycobacterium tuberculosis 19-kilodalton lipoprotein inhibits gamma interferon-regulated HLA-DR and Fc gamma R1 on human macrophages through Toll-like receptor 2. Infect. Immun. 2003, 71, 4487-4497. [CrossRef]

29. Jiang, Y.; Wei, J.; Liu, H.; Li, G.; Guo, Q.; Qiu, Y.; Zhao, L.; Li, M.; Zhao, X.; Dou, X.; et al. Polymorphisms in the PE35 and PPE68 antigens in Mycobacterium tuberculosis strains may affect strain virulence and reflect ongoing immune evasion. Mol. Med. Rep. 2016, 13, 947-954. [CrossRef]

30. Arnvig, K.B.; Comas, I.; Thomson, N.; Houghton, J.; Boshoff, H.I.; Croucher, N.; Rose, G.; Perkins, T.T.; Parkhill, J.; Dougan, G.; et al. Sequence-Based Analysis Uncovers an Abundance of Non-Coding RNA in the Total Transcriptome of Mycobacterium tuberculosis. PLoS Pathog. 2011, 7, e1002342. [CrossRef]

31. Ignatov, D.V.; Salina, E.G.; Fursov, M.V.; Skvortsov, T.A.; Azhikina, T.L.; Kaprelyants, A.S. Dormant non-culturable Mycobacterium tuberculosis retains stable low-abundant mRNA. BMC Genom. 2015, 16, 954. [CrossRef] [PubMed]

32. Pasula, R.; Downing, J.F.; Wright, J.R.; Kachel, D.L.; Davis, T.E., Jr.; Martin, W.J. Surfactant protein A (SP-A) mediates attachment of Mycobacterium tuberculosis to murine alveolar macrophages. Am. J. Respir. Cell Mol. Biol. 1997, 17, 209-217. [CrossRef] [PubMed]

33. Gaynor, C.D.; McCormack, F.; Voelker, D.R.; McGowan, S.; Schlesinger, L.S. Pulmonary surfactant protein A mediates enhanced phagocytosis of Mycobacterium tuberculosis by a direct interaction with human macrophages. J. Immunol. 1995, 155, 5343-5351.

34. Shepherd, V.L.; Lopez, J.P. The Role of Surfactant-Associated Protein A in Pulmonary Host Defense. Immunol. Res. 2001, 23, 111-120. [CrossRef] 
35. Pasula, R.; Wright, J.R.; Kachel, D.L.; Martin, W.J. Surfactant protein A suppresses reactive nitrogen intermediates by alveolar macrophages in response to Mycobacterium tuberculosis. J. Clin. Investig. 1999, 103, 483-490. [CrossRef] [PubMed]

36. Shepelkova, G.; Pommerenke, C.; Alberts, R.; Geffers, R.; Evstifeev, V.; Apt, A.; Schughart, K.; Wilk, E. Analysis of the lung transcriptome in Mycobacterium tuberculosis-infected mice reveals major differences in immune response pathways between TB-susceptible and resistant hosts. Tuberculosis 2013, 93, 263-269. [CrossRef] [PubMed]

37. Shafiani, S.; Tucker-Heard, G.; Kariyone, A.; Takatsu, K.; Urdahl, K.B. Pathogen-specific regulatory T cells delay the arrival of effector T cells in the lung during early tuberculosis. J. Exp. Med. 2010, 207, 1409-1420. [CrossRef]

38. Ulrichs, T.; Kaufmann, S.H. New insights into the function of granulomas in human tuberculosis. J. Pathol. 2005, 208, 261-269. [CrossRef]

39. Malardo, T.; Gardinassi, L.G.; Moreira, B.P.; Padilha, É.; Lorenzi, J.C.C.; Soares, L.S.; Gembre, A.F.; Fontoura, I.C.; De Almeida, L.P.; Santos, I.K.F.d.M.; et al. MicroRNA expression signatures in lungs of mice infected with Mycobacterium tuberculosis. Tuberculosis 2016, 101, 151-159. [CrossRef]

40. Ghorpade, D.S.; Leyland, R.; Kurowska-Stolarska, M.; Patil, S.A.; Balaji, K.N. MicroRNA-155 Is Required for Mycobacterium bovis BCG-Mediated Apoptosis of Macrophages. Mol. Cell. Biol. 2012, 32, 2239-2253. [CrossRef]

41. Rajaram, M.; Ni, B.; Morris, J.D.; Brooks, M.N.; Carlson, T.K.; Bakthavachalu, B.; Schoenberg, D.; Torrelles, J.B.; Schlesinger, L.S. Mycobacterium tuberculosis lipomannan blocks TNF biosynthesis by regulating macrophage MAPK-activated protein kinase 2 (MK2) and microRNA miR-125b. Proc. Natl. Acad. Sci. USA 2011, 108, 17408-17413. [CrossRef]

42. Kumar, R.; Halder, P.; Sahu, S.K.; Kumar, M.; Kumari, M.; Jana, K.; Ghosh, Z.; Sharma, P.; Kundu, M.; Basu, J. Identification of a novel role of ESAT-6-dependent miR-155 induction during infection of macrophages withMycobacterium tuberculosis. Cell. Microbiol. 2012, 14, 1620-1631. [CrossRef]

43. Wang, J.; Yang, K.; Zhou, L.; Wu, M.; Wu, Y.; Zhu, M.; Lai, X.; Chen, T.; Feng, L.; Li, M.; et al. MicroRNA-155 Promotes Autophagy to Eliminate Intracellular Mycobacteria by Targeting Rheb. PLoS Pathog. 2013, 9, e1003697. [CrossRef]

44. Iwai, H.; Funatogawa, K.; Matsumura, K.; Kato-Miyazawa, M.; Kirikae, F.; Kiga, K.; Sasakawa, C.; Miyoshi-Akiyama, T.; Kirikae, T. MicroRNA-155 knockout mice are susceptible to Mycobacterium tuberculosis infection. Tuberculosis 2015, 95, 246-250. [CrossRef] 